\title{
Reduced-basis output bound methods for parabolic problems
}

\author{
D.V. Rovas; L. Machiels† Y. Maday ${ }^{\ddagger}$ \\ Date: October 19, 2004
}

\begin{abstract}
In this paper, we extend reduced-basis output bound methods developed earlier for elliptic problems, to problems described by parametrized parabolic partial differential equations. The essential new ingredient and the novelty of this paper consist in the presence of time in the formulation and solution of the problem. First, without assuming a time discretization, a reduced-basis procedure is presented to efficiently compute accurate approximations to the solution of the parabolic problem and relevant outputs of interest. In addition, we develop an error estimation procedure to a posteriori validate the accuracy of our output predictions. Second, using the discontinuous Galerkin method for the temporal discretization, the reduced-basis method and the output bound procedure are analyzed for the semidiscrete case. In both cases the reduced-basis is constructed by taking snapshots of the solution in both time and parameters. In that sense the method is close to POD.
\end{abstract}

\section{Introduction}

The repeated solution of parametrized partial differential equations for many different input parameters is interesting in many different contexts. The "input" parameters, which we will collectively denote as "inputs," serve to characterize a configuration of the physical system. These inputs may represent design or decision variables, such as geometry or physical properties - for example, in optimization studies; control variables, such as actuator power - for example in real-time applications; or characterization variables, such as physical properties - for example in inverse problems. Of interest is, for a given set of "inputs", to compute the solution of the partial differential equation and, usually, evaluate relevant "outputs" which are performance indicators for the particular configuration - for example, maximum temperatures, stresses, flow-rates. Typically in the contexts above, a repeated solution of the underlying partial differential equations is required - for many different inputs - and this is usually prohibitively expensive.

Especially for parabolic systems, to reduce the computational complexity, model-order reduction techniques have been extensively studied in the literature. Probably the most notable approaches are those based on the proper orthogonal decomposition [7, 10] or the use of Krylov subspaces [4]. In developing such methods a number of issues have to be addressed: we mention here, efficiency of the low-order models, accuracy of their predictions, stability and scalability of the numerical procedures; these, often conflicting, requirements have led to an abundance of methods. Usually relevant a priori results establish the approximation properties of these methods (see [8] for a discussion); on the contrary, ways to a posteriori validate the accuracy are scarce. Moreover, earlier research has focused on developing efficient low-order models that preserve the relevant dynamics of a specific system; with the exception of Balmes [2], the case of parametrized partial differential equations has not be considered.

To address some of these issues, reduced-basis output bound methods have been developed for problems described by elliptic partial differential equations - see [16] for a review of earlier work. In the reduced-basis

\footnotetext{
* Department of Mechanical and Industrial Engineering, University of Illinois at Urbana-Champaign, 332E Mechanical Engineering Building, MC-244, 1206 West Green Street, Urbana, IL, 61801, USA. E-mail: rovas@uiuc.edu

${ }^{\dagger}$ McKinsey Corporation, Brussels, Belgium. E-mail: luc_machiels@mckinsey.com

${ }^{\ddagger}$ Laboratoire Jacques-Louis Lions, Université Pierre et Marie Curie, Boîte courrier 187, 75252 Paris Cedex 05, France. E-mail: maday@ann.jussieu.fr
} 
method $[1,12,15]$ the solution is sought in low-dimensional spaces, comprised of solutions to the original problem for different "inputs" $[2,11]$. The critical observation is that the solution and the outputs evolve in low-dimensional manifolds induced by the parametric dependence of the problem; using these problemspecific spaces we achieve good accuracy at a minimal cost - this is confirmed both in theory [13, 18] and practice [16]. The increased efficiency incurred by this computational relaxation comes at the cost of higher uncertainty. To validate the accuracy of the predictions, a posteriori error estimation techniques have been developed to quantify the error directly for the outputs of interest.

In this paper, we consider the extension of reduced-basis output bound methods developed earlier, to problems described by parabolic partial differential equations. The essential new ingredient in the parabolic case is the presence of time in the formulation and solution of the problem. For the parametrization of the problem, time is considered as an additional parameter, albeit a special one as we will see in the development to follow. For the numerical solution of the problem the finite-element method is employed for the spatial discretization. For the temporal discretization the discontinuous Galerkin method [6,21] is used; although not the only choice, the variational origin of the discontinuous Galerkin is desirable for the development and proof of the bounding properties. A procedure to efficiently calculate upper and lower estimators to the outputs of interest is developed. We prove that these estimators are bounds to the exact value for the output. These bounds can be calculated efficiently by assuming an (often-satisfied) form for the partial differential operator [12]; the details specific to the parabolic case are presented in [19].

\section{Problem Statement}

To start, consider a bounded open domain $\Omega \subset \mathbb{R}^{d}, d=1,2,3$ with Lipschitz-continuous boundary; if $T>0$ is the final time and $I=(0, T)(\bar{I}=[0, T])$ the time interval of interest, we define the "space-time" domain $Q_{T}=I \times \Omega$. Furthermore, let $V$ be a closed linear subspace of $H^{1}(\Omega)$, such that $H_{0}^{1}(\Omega) \subset V \subset H^{1}(\Omega)$. The space $L^{2}(I ; V)$ can be defined as

$$
L^{2}(I ; V)=\left\{v: I \rightarrow V \mid v \text { is measurable and } \int_{I}\|v(t)\|_{1}^{2} d t<\infty\right\} ;
$$

with $\|\cdot\|_{1}$ the usual $H^{1}(\Omega)$-norm. Similarly, we define $C^{0}\left(\bar{I} ; L^{2}(\Omega)\right)$ the set of functions which are continuous (and therefore bounded) in time, and $L^{2}(\Omega)$ in space for $t \in \bar{I}$; also, we will use in the following $L^{2}\left(Q_{T}\right) \equiv$ $L^{2}\left(I ; L^{2}(\Omega)\right)$, and $\mathcal{H} \equiv L^{2}(I ; V) \cap C^{0}\left(\bar{I} ; L^{2}(\Omega)\right)[9,17]$. For the parametric dependence, let $P$ be the number of input parameters and $\mathcal{D} \subset \mathbb{R}^{P}$ the set of allowed configurations; a particular configuration will be denoted by $\mu \in \mathcal{D}$.

Let $f(\cdot ; \mu) \in L^{2}\left(Q_{T}\right)$ and $u_{0}(\mu) \in L^{2}(\Omega)$ be known functions which depend on the parameter $\mu$. The problem we are interested in solving is: given a $\mu \in \mathcal{D}$, find the solution $u(\cdot ; \mu) \in \mathcal{H}$ to the equation:

$$
\begin{gathered}
\left(\partial_{t} u(t ; \mu), v\right)+a(u(t ; \mu), v ; \mu)=(f(t ; \mu), v), \forall v \in V, \\
u(0 ; \mu)=u_{0}(\mu) ;
\end{gathered}
$$

here $(\cdot, \cdot)$ denotes the $L^{2}(\Omega)$-inner product and $a(\cdot, \cdot ; \mu): V \times V \rightarrow \mathbb{R}$ is a continuous and coercive-in- $V$ bilinear form, uniformly in $\mu \in \mathcal{D}$. Equation (1) has to be understood in the proper distributional sense for $t \in I$. Under the assumptions above the problem is parabolic and a unique solution $u(\cdot ; \mu) \in \mathcal{H}$ exists for all $\mu \in \mathcal{D}$ [17]. We should also mention that a solution to (1) exists under weaker assumptions than the ones presented above (e.g. $f(\cdot ; \mu) \in L^{2}\left(I ; V^{\prime}\right)$, with $V^{\prime}$ the dual of $\left.V\right)$ - this generality is not required for our presentation. Also to keep the notation minimal, we assume that the $L^{2}(\Omega)$-inner product and the bilinear form $a(\cdot, \cdot ; \mu)$ do not depend on time.

As was mentioned in Section 1, in practical applications the solution field $u(\cdot ; \mu)$ is less important than relevant outputs of interest. We consider here the output of interest which is obtained from $s(\mu) \equiv \mathcal{S}(u(\cdot ; \mu))$, with $\mathcal{S}: \mathcal{H} \rightarrow \mathbb{R}$ a linear functional

$$
\mathcal{S}(v)=\int_{I}\left(\ell^{O}(t), v(t)\right) d t+\left(g^{O}, v\left(T^{-}\right)\right)
$$


with $v\left(t^{ \pm}\right)=\lim _{s \rightarrow 0^{+}} v\left(t \pm s\right.$ ). Here $\ell^{O}(\cdot) \in L^{2}\left(Q_{T}\right)$ (or more generally, $\ell^{O} \in L^{2}\left(I ; V^{\prime}\right)$ ) and $g^{O} \in L^{2}(\Omega)$ do not depend on $\mu$ - a parametric dependence of the output can be readily treated, see [19] for more details.

It will be useful in the following to replace (1), with a space-time weak formulation: given $\mu \in \mathcal{D}$, find $u(\cdot ; \mu) \in \mathcal{H}$ such that

$$
\int_{I}\left(\partial_{t} u(t ; \mu), v(t)\right) d t+\int_{I} a(u(t ; \mu), v(t) ; \mu) d t+\left(u\left(0^{+} ; \mu\right), v\left(0^{+}\right)\right)=\int_{I}(f(t ; \mu), v(t)) d t+\left(u_{0}(\mu), v\left(0^{+}\right)\right),
$$

$\forall v \in \mathcal{H}$. It is obvious that if $u(\cdot ; \mu)$ is the solution of (1) then it is also a solution of (2). We can readily prove the following:

Lemma 2.1. The problem in (2) is stable, and therefore $u(\cdot ; \mu) \in \mathcal{H}$ is the unique weak solution to (2).

Proof. Stability and therefore uniqueness, follows from the coercivity of the bilinear form $a(\cdot, \cdot ; \mu)$,

$$
\exists \underline{c}>0 \text { such that } \underline{c}\|v\|_{1} \leq a(v, v ; \mu), \forall v \in V, \forall \mu \in \mathcal{D} \text {; }
$$

which implies that

$$
\begin{aligned}
& \int_{I}\left(\partial_{t} v(t), v(t)\right) d t+\int_{I} a(v(t), v(t) ; \mu) d t+\left(v\left(0^{+}\right), v\left(0^{+}\right)\right)= \\
& \frac{1}{2}\left(v\left(T^{-}\right), v\left(T^{-}\right)\right)+\frac{1}{2}\left(v\left(0^{+}\right), v\left(0^{+}\right)\right)+\int_{I} a(v(t), v(t) ; \mu) d t \geq \underline{c}\|v\|_{L^{2}\left(I ; H^{1}\right)}^{2}, \forall v \in \mathcal{H}, v \neq 0 .
\end{aligned}
$$

We will also require in the following $\psi(\cdot ; \mu) \in \mathcal{H}$ which is the solution of the following dual problem:

$$
\begin{aligned}
& -\int_{I}\left(\partial_{t} \psi(t ; \mu), v(t)\right) d t+\int_{I} a(v(t), \psi(t ; \mu) ; \mu) d t+\left(\psi\left(T^{-} ; \mu\right), v\left(T^{-}\right)\right)= \\
& \quad-\int_{I}\left(\ell^{O}(t), v(t)\right) d t-\left(g^{O}, v\left(T^{-}\right)\right), \forall v \in \mathcal{H} ;
\end{aligned}
$$

the importance of the dual problem will become clear in the analysis that follows. Notice that if we define $\tau=T-t$, (3) is actually parabolic: the dual problem evolves backward in time. Therefore, under the requirements above for the primal problem a unique weak solution $\psi(\cdot ; \mu)$ to $(3)$ will exist.

In practice, for the solution of (2) and (3), we replace $V$ by a finite but high-dimensional finite-element space $V_{h}$, so that $V_{h} \approx V\left(\operatorname{dim} V_{h}=\mathcal{N}\right)$. Given an input configuration $\mu$, solution of the resulting system of ordinary differential equations (and relatedly, calculation of the output of interest), can be very expensive. We develop in the next section, a reduced-basis approach to significantly reduce the complexity of this problem.

\section{Reduced-basis Approximation}

We define $\tilde{\mu}=(t, \mu) \in \tilde{\mathcal{D}} \equiv I \times \mathcal{D}$, and introduce the following sample sets $S_{N}^{\mathrm{pr}}=\left\{\tilde{\mu}_{1}^{\mathrm{pr}}, \ldots, \tilde{\mu}_{N}^{\mathrm{pr}}\right\}$ and $S_{M}^{\mathrm{du}}=\left\{\tilde{\mu}_{1}^{\mathrm{du}}, \ldots, \tilde{\mu}_{M}^{\mathrm{du}}\right\}$. In general, $N \neq M$ and $\tilde{\mu}_{i}^{\mathrm{pr}} \neq \tilde{\mu}_{j}^{\mathrm{du}}, i=1, \ldots, N, j=1, \ldots, M$. We then compute the solution of (2) for all $\left\{\mu \in I \mid \exists t:(t, \mu) \in S_{N}^{\mathrm{pr}}\right\}$, and of (3) for all $\left\{\mu \in I \mid \exists t:(t, \mu) \in S_{M}^{\mathrm{du}}\right\}$. Using these solutions we define the Lagrangian reduced-basis approximation spaces, as follows:

$$
W_{N}^{\mathrm{pr}}=\operatorname{span}\left\{\zeta_{i} \equiv u\left(\tilde{\mu}_{i}^{\mathrm{pr}}\right), i=1, \ldots, N\right\}, W_{M}^{\mathrm{du}}=\operatorname{span}\left\{\xi_{i} \equiv \psi\left(\tilde{\mu}_{i}^{\mathrm{du}}\right), i=1, \ldots, M\right\},
$$

where $\operatorname{dim} W_{N}^{\mathrm{pr}}=N$, and $\operatorname{dim} W_{M}^{\mathrm{du}}=M$; by construction $W_{N}^{\mathrm{pr}}, W_{M}^{\mathrm{du}} \subset V$. We can then define the following spaces,

$$
\mathcal{H}_{N}^{\mathrm{pr}} \equiv L^{2}\left(I ; W_{N}^{\mathrm{pr}}\right) \cap C^{0}\left(\bar{I} ; L^{2}(\Omega)\right), \text { and } \mathcal{H}_{M}^{\mathrm{du}} \equiv L^{2}\left(I ; W_{M}^{\mathrm{du}}\right) \cap C^{0}\left(\bar{I} ; L^{2}(\Omega)\right) .
$$


In the construction of the reduced-basis spaces the choice of $\mu_{i} \in \mathcal{D}$ (and consequently $\tilde{\mu}_{i}$ ) for the sample sets $S_{N}^{\mathrm{pr}}$ and $S_{M}^{\mathrm{du}}$ is critical. Both the a priori theory [13] (in the context of elliptic problems) and extensive numerical tests $[16,19]$ suggest that the points should be chosen "log-randomly" over $\mathcal{D}$ : we sample from a multivariate uniform probability density on $\log (\mathcal{D})$. Especially for large ranges of the input parameters, this logarithmic distribution performs considerably better than other obvious candidates.

The reduced-basis approximation $u_{N}(\cdot ; \mu)$ to $u(\cdot ; \mu)$ is obtained by a standard Galerkin projection: given a $\mu \in \mathcal{D}$, find $u_{N}(\cdot ; \mu) \in \mathcal{H}_{N}^{\mathrm{pr}}$, such that

$$
\begin{aligned}
\int_{I}\left(\partial_{t} u_{N}(t ; \mu), v(t)\right) d t+\int_{I} a\left(u_{N}(t ; \mu), v(t) ; \mu\right) d t+\left(u_{N}\left(0^{+} ; \mu\right), v\left(0^{+}\right)\right)= & \\
& \int_{I}(f(t ; \mu), v(t)) d t+\left(u_{0}(\mu), v\left(0^{+}\right)\right), \forall v \in \mathcal{H}_{N}^{\mathrm{pr}} .
\end{aligned}
$$

The error to the approximation of $u(\cdot ; \mu)$ by $u_{N}(\cdot ; \mu)$ is $e^{\operatorname{pr}}(t ; \mu) \equiv u(t ; \mu)-u_{N}(t ; \mu)$, and relatedly $\mathcal{R}^{\text {pr }}(v ; \mu)$ is the residual for the primal problem:

$$
\begin{aligned}
& \mathcal{R}^{\mathrm{pr}}(v ; \mu)= \\
& =\int_{I}(f(t ; \mu), v(t)) d t-\int_{I}\left(\partial_{t} u_{N}(t ; \mu), v(t)\right) d t-\int_{I} a\left(u_{N}(t ; \mu), v(t) ; \mu\right) d t-\left(u_{N}\left(0^{+} ; \mu\right)-u_{0}(\mu), v\left(0^{+}\right)\right) \\
& =\int_{I}\left(\partial_{t} e^{\mathrm{pr}}(t ; \mu), v(t)\right) d t+\int_{I} a\left(e^{\mathrm{pr}}(t ; \mu), v(t) ; \mu\right) d t+\left(e^{\mathrm{pr}}\left(0^{+} ; \mu\right), v\left(0^{+}\right)\right)
\end{aligned}
$$

the last line above follows from (2). Similarly, for the dual variable, we obtain an approximation $\psi_{M}(\cdot ; \mu) \in$ $\mathcal{H}_{M}^{\mathrm{du}}$ to $\psi(\cdot ; \mu) \in \mathcal{H}$ from:

$$
\begin{aligned}
-\int_{I}\left(\partial_{t} \psi_{M}(t ; \mu), v(t)\right) d t+\int_{I} a\left(v(t), \psi_{M}(t ; \mu) ; \mu\right) d t & +\left(\psi_{M}\left(T^{-} ; \mu\right), v\left(T^{-}\right)\right)= \\
& -\int_{I}\left(\ell^{O}(t), v(t)\right) d t-\left(g^{O}, v\left(T^{-}\right)\right), \forall v \in \mathcal{H}_{M}^{\mathrm{du}} .
\end{aligned}
$$

The residual for the dual problem $\mathcal{R}^{\mathrm{du}}(v ; \mu)$ is then:

$$
\begin{aligned}
& \mathcal{R}^{\mathrm{du}}(v ; \mu)= \\
& =-\int_{I}\left(\ell^{O}(t), v(t)\right) d t+\int_{I}\left(\partial_{t} \psi_{M}(t ; \mu), v(t)\right) d t-\int_{I} a\left(v(t), \psi_{M}(t ; \mu) ; \mu\right) d t-\left(\psi_{M}\left(T^{-} ; \mu\right)+g^{O}, v\left(T^{-}\right)\right) \\
& =-\int_{I}\left(\partial_{t} e^{\mathrm{du}}(t ; \mu), v(t)\right) d t+\int_{I} a\left(v(t), e^{\mathrm{du}}(t ; \mu) ; \mu\right) d t+\left(e^{\mathrm{du}}\left(T^{-} ; \mu\right), v\left(T^{-}\right)\right)
\end{aligned}
$$

from (3) and defining $e^{\mathrm{du}}(t ; \mu)=\psi(t ; \mu)-\psi_{M}(t ; \mu)$.

Using now the reduced-basis solutions to the primal and dual problems, we can obtain an approximation to the output of interest $s_{N}(\mu)$ from:

$$
s_{N}(\mu) \equiv \mathcal{S}\left(u_{N}(\cdot ; \mu)\right)-\mathcal{R}^{\mathrm{pr}}\left(\psi_{M}(\cdot ; \mu) ; \mu\right)=\int_{I}\left(\ell^{O}(t), u_{N}(t ; \mu)\right) d t+\left(g^{O}, u_{N}\left(T^{-} ; \mu\right)\right)-\mathcal{R}^{\mathrm{pr}}\left(\psi_{M}(\cdot ; \mu) ; \mu\right) .
$$

Regarding the convergence of the output approximation (8), we have the following:

Lemma 3.1. Let

$$
\begin{aligned}
\varepsilon_{M}^{\mathrm{du}}=\inf _{\chi_{M} \in \mathcal{H}_{M}^{\mathrm{du}}}\left\{\left[\left\|e^{\mathrm{pr}}\right\|_{L^{\infty}\left(I ; L^{2}\right)}+\left\|e^{\mathrm{pr}}\right\|_{L^{2}\left(I ; H^{1}\right)}\right] \times\left[\left\|\psi-\chi_{M}\right\|_{L^{\infty}\left(I ; L^{2}\right)}+\left\|\psi-\chi_{M}\right\|_{L^{2}\left(I ; H^{1}\right)}\right]\right. \\
\left.+\left\|e^{\mathrm{pr}}\right\|_{L^{2}\left(I ; L^{2}\right)}\left\|\psi-\chi_{M}\right\|_{H^{1}\left(I ; L^{2}\right)}\right\}
\end{aligned}
$$

then

$$
\left|s(\mu)-s_{N}(\mu)\right| \leq C\left[\left\|e^{\mathrm{pr}}\right\|_{L^{\infty}\left(I ; L^{2}\right)}+\left\|e^{\mathrm{pr}}\right\|_{L^{2}\left(I ; H^{1}\right)}\right] \times\left[\left\|e^{\mathrm{du}}\right\|_{L^{\infty}\left(I ; L^{2}\right)}+\left\|e^{\mathrm{du}}\right\|_{L^{2}\left(I ; H^{1}\right)}\right]+C \varepsilon_{M}^{\mathrm{du}} .
$$


Proof. We start with an auxiliary result that will also be required below,

$$
\begin{aligned}
s(\mu)-s_{N}(\mu) & =\int_{I}\left(\ell^{O}, u\right) d t+\left(g^{O}, u\left(T^{-}\right)\right)-\int_{I}\left(\ell^{O}, u_{N}\right) d t-\left(g^{O}, u_{N}\left(T^{-}\right)\right)+\mathcal{R}^{\mathrm{pr}}\left(\psi_{M} ; \mu\right) \\
& =\int_{I}\left(\ell^{O}, e^{\mathrm{pr}}\right) d t+\left(g^{O}, e^{\mathrm{pr}}\left(T^{-}\right)\right)+\mathcal{R}^{\mathrm{pr}}\left(\psi_{M} ; \mu\right) \\
& =\int_{I}\left(\partial_{t} \psi, e^{\mathrm{pr}}\right) d t-\int_{I} a\left(e^{\mathrm{pr}}, \psi ; \mu\right) d t-\left(\psi\left(T^{-}\right), e^{\mathrm{pr}}\left(T^{-}\right)\right)+\mathcal{R}^{\mathrm{pr}}\left(\psi_{M} ; \mu\right) \\
& =-\int_{I}\left(\partial_{t} e^{\mathrm{pr}}, \psi\right) d t-\int_{I} a\left(e^{\mathrm{pr}}, \psi ; \mu\right) d t-\left(e^{\mathrm{pr}}\left(0^{+}\right), \psi\left(0^{+}\right)\right)+\mathcal{R}^{\mathrm{pr}}\left(\psi_{M} ; \mu\right) \\
& =-\mathcal{R}^{\mathrm{pr}}\left(e^{\mathrm{du}} ; \mu\right) ;
\end{aligned}
$$

using (3), integration by parts, (5) and linearity of the primal residual. From (10)

$$
\left|s(\mu)-s_{N}(\mu)\right|=\left|\int_{I}\left(\partial_{t} e^{\mathrm{pr}}, e^{\mathrm{du}}\right) d t+\int_{I} a\left(e^{\mathrm{pr}}, e^{\mathrm{du}} ; \mu\right) d t+\left(e^{\mathrm{pr}}(0), e^{\mathrm{du}}(0)\right)\right| ;
$$

we look at each of the terms on the right-hand side separately. For any $\chi_{M}(\cdot) \in \mathcal{H}_{M}^{\mathrm{du}}$, then

$$
\begin{aligned}
\left|\int_{I}\left(\partial_{t} e^{\mathrm{pr}}, e^{\mathrm{du}}\right) d t\right| & =\left|\int_{I}\left(\partial_{t} e^{\mathrm{pr}}, \psi-\chi_{M}+\chi_{M}-\psi_{M}\right) d t\right| \\
& \leq\left|\int_{I}\left(\partial_{t} e^{\mathrm{pr}}, \psi-\chi_{M}\right) d t\right|+\left|\int_{I}\left(\partial_{t} e^{\mathrm{pr}}, \psi_{M}-\chi_{M}\right) d t\right|
\end{aligned}
$$

For the first term above we use integration by parts to get:

$$
\left|\int_{I}\left(\partial_{t} e^{\mathrm{pr}}, \psi-\chi_{M}\right) d t\right| \leq C\left[\left\|e^{\mathrm{pr}}\right\|_{L^{\infty}\left(I ; L^{2}\right)}\left\|\psi-\chi_{M}\right\|_{L^{\infty}\left(I ; L^{2}\right)}+\left\|e^{\mathrm{pr}}\right\|_{L^{2}\left(I ; L^{2}\right)}\left\|\psi-\chi_{M}\right\|_{H^{1}\left(I ; L^{2}\right)}\right] ;
$$

and for the second term from (5) and using the Galerkin orthogonality property (since $\left.\psi_{M}(t)-\chi_{M}(t) \in W_{M}^{\mathrm{du}}\right)$, we get:

$$
\begin{aligned}
\left|\int_{I}\left(\partial_{t} e^{\mathrm{pr}}, \psi_{M}-\chi_{M}\right) d t\right|= & \left|\int_{I} a\left(e^{\mathrm{pr}}, \psi_{M}-\chi_{M} ; \mu\right) d t+\left(e^{\mathrm{pr}}\left(0^{+}\right), \psi_{M}\left(0^{+}\right)-\chi_{M}\left(0^{+}\right)\right)\right| \\
\leq & \gamma\left\|e^{\mathrm{pr}}\right\|_{L^{2}\left(I ; H^{1}\right)}\left\|\psi_{M}-\psi+\psi-\chi_{M}\right\|_{L^{2}\left(I ; H^{1}\right)} \\
& +\left\|e^{\mathrm{pr}}\right\|_{L^{\infty}\left(I ; L^{2}\right)}\left[\left\|\psi_{M}-\psi\right\|_{L^{\infty}\left(I ; L^{2}\right)}+\left\|\psi-\chi_{M}\right\|_{L^{\infty}\left(I ; L^{2}\right)}\right] \\
\leq & \gamma\left\|e^{\mathrm{pr}}\right\|_{L^{2}\left(I ; H^{1}\right)}\left(\left\|\psi_{M}-\psi\right\|_{L^{2}\left(I ; H^{1}\right)}+\left\|\psi-\chi_{M}\right\|_{L^{2}\left(I ; H^{1}\right)}\right) \\
& +\left\|e^{\mathrm{pr}}\right\|_{L^{\infty}\left(I ; L^{2}\right)}\left\|e^{\mathrm{du}}\right\|_{L^{\infty}\left(I ; L^{2}\right)}+\left\|e^{\mathrm{pr}}\right\|_{L^{\infty}\left(I ; L^{2}\right)}\left\|\psi-\chi_{M}\right\|_{L^{\infty}\left(I ; L^{2}\right)},
\end{aligned}
$$

with $\gamma$ the continuity constant of $a(\cdot, \cdot ; \mu)$. Combining the expressions above and minimizing over all $\chi_{M}$ yield

$$
\left|\int_{I}\left(\partial_{t} e^{\mathrm{pr}}, e^{\mathrm{du}}\right) d t\right| \leq \gamma\left\|e^{\mathrm{pr}}\right\|_{L^{2}\left(I ; H^{1}\right)}\left\|e^{\mathrm{du}}\right\|_{L^{2}\left(I ; H^{1}\right)}+C \varepsilon_{M}^{\mathrm{du}} .
$$

The second and third terms in (11) can be bounded using the continuity of the bilinear form $a$ and the Cauchy-Schwartz inequality, giving

$$
\left|s(\mu)-s_{N}(\mu)\right| \leq\left|\int_{I}\left(\partial_{t} e^{\mathrm{pr}}, e^{\mathrm{du}}\right) d t\right|+\gamma\left\|e^{\mathrm{pr}}\right\|_{L^{2}\left(I ; H^{1}\right)}\left\|e^{\mathrm{du}}\right\|_{L^{2}\left(I ; H^{1}\right)}+\left\|e^{\mathrm{pr}}\right\|_{L^{\infty}\left(I ; L^{2}\right)}\left\|e^{\mathrm{du}}\right\|_{L^{\infty}\left(I ; L^{2}\right)} .
$$

The desired result follows directly from (12) and (13). 
The previous lemma gives an a priori bound on the convergence of the output approximation, defined in (8), to its exact value; as we see from (9), a term appears involving $\varepsilon_{M}^{\mathrm{du}}$ - a measure of how well members of the reduced-basis space $\mathcal{H}_{M}^{\mathrm{du}}$ approximate the solution to the adjoint problem - as well as norms of the error to the dual problem $e^{\mathrm{du}}$. Had we used $\mathcal{S}\left(u_{N}(\cdot ; \mu)\right)$ instead of (8) to calculate the output approximation, the corresponding bound would depend on norms of the primal error $e^{\text {pr }}$ only. As $M$ increases, the term involving the dual errors will become smaller and, given the approximation properties of $W_{M}^{\mathrm{du}}$, will converge to zero; this suggests faster convergence of the adjoint-corrected output and use of (8) is justified.

In the calculation above we have, in effect, replaced $V$ (or $V_{h}$ ) with $W_{N}^{\mathrm{pr}}$ for the primal and $W_{M}^{\mathrm{du}}$ for the dual problem. These reduced-basis spaces have approximation properties specific to the problem of interest, so only a small number of basis functions need to be retained to accurately represent the solution. Significant computational savings are affected, since the computational complexity scales as $N\left(=\operatorname{dim} W_{N}^{\text {pr }}\right)$ and $M\left(=\operatorname{dim} W_{M}^{\mathrm{du}}\right)$ instead of $\mathcal{N}\left(=\operatorname{dim} V_{h}\right)$, and $N, M$ will be small — typically $O(10)$ - and independent of $\mathcal{N}$. As $N, M \rightarrow \infty$, and given the specific choice of the approximation spaces, $u_{N}(\cdot ; \mu) \rightarrow u(\cdot ; \mu)$, $\psi_{M}(\cdot ; \mu) \rightarrow \psi(\cdot ; \mu)$, and $s_{N}(\mu) \rightarrow s(\mu)$ will converge to the exact values very fast.

\section{A posteriori error estimation}

The computational relaxation introduced in the previous section, allows us to compute very efficiently accurate approximations to the solution and the output of interest. Thanks to the expected rapid convergence $N$ and $M$ could, in theory, be chosen quite small. However, in practice we do not know how small $N$ and $M$ can be: this will depend of the desired accuracy, the choice of $\tilde{\mu}_{i}$ in the construction of the reduced-basis spaces, the output of interest and the particular problem in question; in some cases $N, M=5$ may suffice, while in other cases $N, M=100$ may still be insufficient. In the face of this uncertainty, either too many or too few basis functions will be retained: the former results in computational inefficiency; the later in unacceptable uncertainty. It is therefore critical that we can ascertain the accuracy of our predictions; we develop next, a rigorous error-estimation approach, directly for outputs of interest, to a posteriori validate the accuracy of our predictions.

To begin assume that we may find a function $g(\mu): \mathcal{D} \rightarrow \mathbb{R}_{+}$, and a symmetric, continuous and coercive bilinear form $\hat{a}: V \times V \rightarrow \mathbb{R}$ such that

$$
\underline{c}\|v\|_{1} \leq g(\mu) \hat{a}(v, v) \leq a(v, v ; \mu), \forall v \in V, \forall \mu \in \mathcal{D} ;
$$

we understand $g(\mu)$ as a lower bound to the $\hat{a}$-coercivity constant.

We then compute the "reconstructed" errors $\hat{e}^{\mathrm{pr}}(\cdot ; \mu) \in \mathcal{H}$ and $\hat{e}^{\mathrm{du}}(\cdot ; \mu) \in \mathcal{H}$ such that

$$
\begin{aligned}
g(\mu) \int_{I} \hat{a}\left(\hat{e}^{\mathrm{pr}}(t ; \mu), v(t)\right) d t & =\mathcal{R}^{\mathrm{pr}}(v ; \mu), \forall v \in \mathcal{H}, \text { and } \\
g(\mu) \int_{I} \hat{a}\left(\hat{e}^{\mathrm{du}}(t ; \mu), v(t)\right) d t & =\mathcal{R}^{\mathrm{du}}(v ; \mu), \forall v \in \mathcal{H} .
\end{aligned}
$$

Note that a unique solution exists for problems (15), by an application of the Riesz-Frechet representation theorem since $\mathcal{R}^{\mathrm{pr}}$ and $\mathcal{R}^{\mathrm{du}}$ are continuous linear functionals on the Hilbert space $L^{2}\left(I ; V^{\prime}\right)$ and $\int_{I} \hat{a}(\cdot, \cdot) d t$ is a scalar product in $L^{2}(I ; V)$. An estimate for the output is then computed, $s_{B}(\mu)$ :

$$
s_{B}(\mu)=s_{N}(\mu)-\frac{g(\mu)}{2} \int_{I} \hat{a}\left(\hat{e}^{\mathrm{pr}}(t ; \mu), \hat{e}^{\mathrm{du}}(t ; \mu)\right) d t
$$

and a bound gap $\Delta(\mu)$ :

$$
\Delta(\mu)=\frac{g(\mu)}{2}\left[\int_{I} \hat{a}\left(\hat{e}^{\mathrm{pr}}(t ; \mu), \hat{e}^{\mathrm{pr}}(t ; \mu)\right) d t\right]^{\frac{1}{2}}\left[\int_{I} \hat{a}\left(\hat{e}^{\mathrm{du}}(t ; \mu), \hat{e}^{\mathrm{du}}(t ; \mu)\right) d t\right]^{\frac{1}{2}} .
$$

Finally, upper and lower output estimators can be calculated from $s^{ \pm}(\mu)=s_{B}(\mu) \pm \Delta(\mu)$. We now prove that these estimators $s^{ \pm}(\mu)$ are always rigorous bounds to the true output $s(\mu)$. In the proof that follows, unless it is essential, we will not explicitly indicate dependence on the variables $t$ and $\mu$. 
Proposition 1. Let $s_{B}(\mu)$ be the output approximation, defined in (16), and $\Delta(\mu)$ the bound gap, defined in (17). If we then define $s^{ \pm}(\mu)=s_{B}(\mu) \pm \Delta(\mu)$ then

$$
s^{-}(\mu) \leq s(\mu) \leq s^{+}(\mu), \forall \mu \in \mathcal{D} ;
$$

that is, $s^{+}(\mu)$ and $s^{-}(\mu)$ are rigorous upper and lower bounds to the true output $s(\mu)$.

Proof. To start, notice that $\mathcal{R}^{\mathrm{pr}}\left(e^{\mathrm{du}} ; \mu\right)=\mathcal{R}^{\mathrm{du}}\left(e^{\mathrm{pr}} ; \mu\right)$ since:

$$
\begin{aligned}
\mathcal{R}^{\mathrm{pr}}\left(e^{\mathrm{du}} ; \mu\right) & =\int_{I}\left(\partial_{t} e^{\mathrm{pr}}, e^{\mathrm{du}}\right) d t+\int_{I} a\left(e^{\mathrm{pr}}, e^{\mathrm{du}} ; \mu\right) d t+\left(e^{\mathrm{pr}}\left(0^{+}\right), e^{\mathrm{du}}\left(0^{+}\right)\right) \\
& =-\int_{I}\left(\partial_{t} e^{\mathrm{du}}, e^{\mathrm{pr}}\right) d t+\int_{I} a\left(e^{\mathrm{pr}}, e^{\mathrm{du}} ; \mu\right) d t+\left(e^{\mathrm{pr}}\left(T^{-}\right), e^{\mathrm{du}}\left(T^{-}\right)\right) \\
& =\mathcal{R}^{\mathrm{du}}\left(e^{\mathrm{pr}} ; \mu\right)
\end{aligned}
$$

using integration by parts, and the definition of the primal (5) and dual (7) residuals. Therefore from (10),

$$
-\mathcal{R}^{\mathrm{du}}\left(e^{\mathrm{pr}} ; \mu\right)=s(\mu)-s_{N}(\mu)
$$

We can now start the proof of the bounding property, and define $\hat{e}^{ \pm}=\hat{e}^{\mathrm{pr}} \mp \frac{1}{\kappa} \hat{e}^{\mathrm{du}}$, with $\kappa>0$. From the coercivity of $\hat{a}$, we have:

$$
\kappa g(\mu) \int_{I} \hat{a}\left(e^{\mathrm{pr}}-\frac{1}{2} \hat{e}^{ \pm}, e^{\mathrm{pr}}-\frac{1}{2} \hat{e}^{ \pm}\right)=\kappa g(\mu) \int_{I} \hat{a}\left(e^{\mathrm{pr}}, e^{\mathrm{pr}}\right)+\kappa \frac{g(\mu)}{4} \int_{I} \hat{a}\left(\hat{e}^{ \pm}, \hat{e}^{ \pm}\right)-\kappa g(\mu) \int_{I} \hat{a}\left(\hat{e}^{ \pm}, e^{\mathrm{pr}}\right) \geq 0 .
$$

Since $\hat{e}^{ \pm}=\hat{e}^{\mathrm{pr}} \mp \frac{1}{\kappa} \hat{e}^{\mathrm{du}}$, and using (15) we get:

$$
g(\mu) \int_{I} \hat{a}\left(\hat{e}^{ \pm}, e^{\mathrm{pr}}\right) d t=\mathcal{R}^{\mathrm{pr}}\left(e^{\mathrm{pr}} ; \mu\right) \mp \frac{1}{\kappa} \mathcal{R}^{\mathrm{du}}\left(e^{\mathrm{pr}} ; \mu\right) .
$$

But:

$$
\begin{aligned}
\mathcal{R}^{\mathrm{pr}}\left(e^{\mathrm{pr}} ; \mu\right) & =\int_{I}\left(\partial_{t} e^{\mathrm{pr}}, e^{\mathrm{pr}}\right) d t+\int_{I} a\left(e^{\mathrm{pr}}, e^{\mathrm{pr}} ; \mu\right) d t+\left(e^{\mathrm{pr}}\left(0^{+}\right), e^{\mathrm{pr}}\left(0^{+}\right)\right) \\
& \geq \frac{1}{2} \underbrace{\left(e^{\mathrm{pr}}\left(T^{-}\right), e^{\mathrm{pr}}\left(T^{-}\right)\right)}_{>0}+\frac{1}{2} \underbrace{\left(e^{\mathrm{pr}}\left(0^{+}\right), e^{\mathrm{pr}}\left(0^{+}\right)\right)}_{>0}+\int_{I} a\left(e^{\mathrm{pr}}, e^{\mathrm{pr}} ; \mu\right) d t \geq g(\mu) \int_{I} \hat{a}\left(e^{\mathrm{pr}}, e^{\mathrm{pr}}\right) d t,
\end{aligned}
$$

since from (14) we have $\int_{I} a\left(e^{\mathrm{pr}}, e^{\mathrm{pr}} ; \mu\right) d t \geq g(\mu) \int_{I} \hat{a}\left(e^{\mathrm{pr}}, e^{\mathrm{pr}}\right) d t$. Replacing in (20) for $\mathcal{R}^{\mathrm{pr}}\left(e^{\mathrm{pr}} ; \mu\right)$ the expression we just obtained, and (18) for $\mathcal{R}^{\mathrm{du}}\left(e^{\mathrm{pr}} ; \mu\right)$, we have:

$$
-\kappa g(\mu) \int_{I} \hat{a}\left(\hat{e}^{ \pm}, e^{\mathrm{pr}}\right) d t \leq-\kappa g(\mu) \int_{I} \hat{a}\left(e^{\mathrm{pr}}, e^{\mathrm{pr}}\right) d t \mp\left(s(\mu)-s_{N}(\mu)\right) .
$$

Combining now (19) and (21), we get

$$
\pm\left(s(\mu)-s_{N}(\mu)\right) \leq \frac{\kappa g(\mu)}{4} \int_{I} \hat{a}\left(\hat{e}^{ \pm}, \hat{e}^{ \pm}\right) d t
$$

Expanding $\hat{e}^{ \pm}=\hat{e}^{\mathrm{pr}} \mp \frac{1}{\kappa} \hat{e}^{\mathrm{du}}$ we have

$$
\pm\left(s(\mu)-s_{N}(\mu)\right) \leq \frac{g(\mu)}{4}\left[\kappa \int_{I} \hat{a}\left(\hat{e}^{\mathrm{pr}}, \hat{e}^{\mathrm{pr}}\right) d t+\frac{1}{\kappa} \int_{I} \hat{a}\left(\hat{e}^{\mathrm{du}}, \hat{e}^{\mathrm{du}}\right) d t \mp 2 \int_{I} \hat{a}\left(\hat{e}^{\mathrm{pr}}, \hat{e}^{\mathrm{du}}\right) d t\right]
$$

and from the definition of $s_{B}(\mu)=s_{N}(\mu)-\frac{g(\mu)}{2} \int_{I} \hat{a}\left(\hat{e}^{\mathrm{pr}}, \hat{e}^{\mathrm{du}}\right) d t$,

$$
\pm\left(s(\mu)-s_{B}(\mu)\right) \leq \frac{\kappa g(\mu)}{4} \int_{I} \hat{a}\left(\hat{e}^{\mathrm{pr}}, \hat{e}^{\mathrm{pr}}\right) d t+\frac{g(\mu)}{4 \kappa} \int_{I} \hat{a}\left(\hat{e}^{\mathrm{du}}, \hat{e}^{\mathrm{du}}\right) d t .
$$


Since $\kappa$ is an arbitrary positive constant, we choose it as:

$$
\kappa=\left(\frac{\int_{I} \hat{a}\left(\hat{e}^{\mathrm{du}}, \hat{e}^{\mathrm{du}}\right) d t}{\int_{I} \hat{a}\left(\hat{e}^{\mathrm{pr}}, \hat{e}^{\mathrm{pr}}\right) d t}\right)^{\frac{1}{2}}
$$

so that the right-hand side in (22) is minimized. Then

$$
\pm\left(s(\mu)-s_{B}(\mu)\right) \leq \frac{g(\mu)}{2}\left[\int_{I} \hat{a}\left(\hat{e}^{\mathrm{pr}}, \hat{e}^{\mathrm{pr}}\right) d t\right]^{\frac{1}{2}}\left[\int_{I} \hat{a}\left(\hat{e}^{\mathrm{du}}, \hat{e}^{\mathrm{du}}\right) d t\right]^{\frac{1}{2}}
$$

which from the definition of $\Delta(\mu)$ becomes $\pm\left(s(\mu)-s_{B}(\mu)\right) \leq \Delta(\mu)$, or

$$
s^{-}(\mu) \equiv s_{B}(\mu)-\Delta(\mu) \leq s(\mu) \leq s_{B}(\mu)+\Delta(\mu) \equiv s^{+}(\mu) .
$$

So following the previous theorem, instead of using the exact value for the output $s(\mu)$, we can use the output prediction $s_{B}(\mu)$ and the bound gap $\Delta(\mu)$. The basic premise is that these two quantities can be computed more efficiently than the exact output. This is indeed the case when a certain decomposition exists for all the parameter-dependent linear and bilinear forms [11, 19]. More specifically, assume that for $t \in I, \mu \in \mathcal{D}$ and for $Q_{a, f, u} \in \mathbb{N}$ the following "affine" decomposition exists:

$$
a(w, v ; \mu)=\sum_{q=1}^{Q_{a}} \sigma_{a}^{q}(\mu) a^{q}(w, v), \forall w, v \in V^{2}, \quad f(t ; \mu)=\sum_{q=1}^{Q_{f}} \sigma_{f}^{q}(t ; \mu) f^{q}, \quad u_{0}(\mu)=\sum_{q=1}^{Q_{u}} \sigma_{u}^{q}(\mu) u_{0}^{q} ;
$$

with $\sigma_{a, f, u}^{q}$ functions which depend on $\mu$ and $t$, whereas the $a^{q}, f^{q}$, and $u_{0}^{q}$ do not. For a large class of problems such a decomposition exists; certain relaxations are possible for locally non-affine problems [16]. For general nonlinear problems, the use of the "empirical interpolation" method [3] is developed in the framework of parabolic problems [5].

Using (23) and following the same steps as in [12], a two-stage computational procedure can be developed: Off-line the reduced-basis space is formed and a database with certain auxiliary quantities is created; this is a relatively expensive preprocessing step which needs to be performed only once. On-line, for each new $\mu$, using the database: the reduced-basis problem is formed and solved; the reduced-basis solution is used to compute the output approximation; and finally, the output bounds are calculated. The incremental cost for each on-line step is minimal and scales only with the dimension $N, M$ of the reduced-basis spaces and the parametric complexity $Q_{a, f, u}$ of the linear and bilinear forms. We do not discuss further the computational procedure here; for more details, specific to the parabolic case, see [19].

The definition of the reduced-basis spaces comprising of snapshots to the solution at different parameter points is not the only possibility. An alternative approach is to construct the reduced-basis spaces by using the entire time-dependent solution at certain parameter values. A space-time Galerkin projection can then be used to obtain the reduced-basis problems. Moreover the a posteriori error estimator, defined above, could be easily adapted to this case. There are certain advantages in this alternative approach; for example, instead of solving the low-dimensional parabolic problems (4) and (6), one has to solve linear systems of small dimension. Also, there is some simplification in the computation of the error estimator. On the other hand, during the preprocessing/off-line stage the computational cost and required memory storage become much higher, making overall this second approach less attractive.

\section{Time Discretization - Discontinuous Galerkin Method}

In the previous section we presented the general theory without any reference to the time-discretization procedure. Here we consider one possible time-discretization method, the discontinuous Galerkin method. 
The discontinuous Galerkin method was first introduced in the context of time-dependent problems by Jamet [6], and was further analyzed [14, 20]. The variational origin of the discontinuous Galerkin method, will allow us to extend the a posteriori error estimation method developed in the previous section for the discrete-in-time approximation.

Consider a set of $L+1$ points in $\bar{I}=[0, T]$ such that $t_{0} \equiv 0<t_{1}<t_{2}<\ldots<t_{L} \equiv T$ is a partition $\mathcal{I}$ of $I$ in intervals $I_{l}=\left(t_{l-1}, t_{l}\right), l \in \mathcal{L} \equiv\{1, \ldots, L\}$. The diameter for each $I_{l}$ will be $\Delta \tau^{l}=t_{l}-t_{l-1}, l \in \mathcal{L}$. We then define the spaces $\mathbb{P}^{q}\left(I_{l} ; V\right)=\left\{v: I_{l} \rightarrow V \mid v(t)=\sum_{s=0}^{q} v_{s} t^{s}, v_{s} \in V\right\} \subset L^{2}\left(I_{l} ; V\right) \cap C^{0}\left(\bar{I}_{l} ; L^{2}(\Omega)\right), \forall l \in \mathcal{L}$, and $V^{q}(\mathcal{I} ; V)=\left\{v \in L^{2}(I ; V)|v|_{I_{l}} \in \mathbb{P}^{q}\left(I_{l} ; V\right), \forall I_{l} \in \mathcal{I}\right\}$. Obviously, if $v \in V^{q}(\mathcal{I} ; V)$ then the function can be discontinuous at the points $t_{l}, l \in \mathcal{L}$. We further define the jump at these points as $[v]_{l}=v\left(t_{l}^{+}\right)-v\left(t_{l}^{-}\right), l \in$ $\{0, \ldots, L\}$, with $v\left(t_{l}^{ \pm}\right)=\lim _{s \rightarrow 0^{+}} v\left(t_{l} \pm s\right)$. The problem is then to compute using the discontinuous Galerkin method a solution $u^{q}(\cdot ; \mu) \in V^{q}(\mathcal{I} ; V)$ - which is a discontinuous approximation to $u(\cdot ; \mu)$ of $(2)$ - from:

$$
\int_{I}\left(\partial_{t} u^{q}(t ; \mu), v(t)\right) d t+\int_{I} a\left(u^{q}(t ; \mu), v(t) ; \mu\right) d t+\sum_{l \in \mathcal{L}}\left(\left[u^{q}(\cdot ; \mu)\right]_{l-1}, v\left(t_{l-1}^{+}\right)\right)=\int_{I}(f(t ; \mu), v(t)) d t,
$$

$\forall v \in V^{q}(\mathcal{I} ; V)$; with $\left[u^{q}(\cdot ; \mu)\right]_{0}=u^{q}\left(0^{+} ; \mu\right)-u_{0}(\mu)$ (or $\left.u^{q}\left(0^{-} ; \mu\right)=u_{0}(\mu)\right)$. In (24) we can solve separately for each $I_{l}$; continuity is imposed only weakly due to the presence of the additional jump terms. For the dual problem, we can compute a solution $\psi^{q}(\cdot ; \mu) \in V^{q}(\mathcal{I} ; V)$ from:

$$
-\int_{I}\left(\partial_{t} \psi^{q}(t ; \mu), v(t)\right) d t+\int_{I} a\left(v(t), \psi^{q}(t ; \mu) ; \mu\right) d t-\sum_{l \in \mathcal{L}}\left(\left[\psi^{q}(\cdot ; \mu)\right]_{l}, v\left(t_{l}^{-}\right)\right)=-\int_{I}\left(\ell^{O}(t), v(t)\right) d t
$$

$\forall v \in V^{q}(\mathcal{I} ; V)$; with $\left[\psi^{q}(\cdot ; \mu)\right]_{L}=-g^{O}-\psi^{q}\left(T^{-} ; \mu\right)$ (or $\left.\psi^{q}\left(T^{+} ; \mu\right)=-g^{O}\right)$. The output of interest $s^{q}(\mu)$ can then be calculated using $u^{q}(\cdot ; \mu)$, from:

$$
s^{q}(\mu)=\int_{I}\left(\ell^{O}(t), u^{q}(t ; \mu)\right) d t+\left(g^{O}, u^{q}\left(T^{-} ; \mu\right)\right)=\sum_{l \in \mathcal{L}} \int_{I_{l}}\left(\ell^{O}(t), u^{q}(t ; \mu)\right) d t+\left(g^{O}, u^{q}\left(T^{-} ; \mu\right)\right) .
$$

The reduced-basis spaces are formed similarly to the continuous case, by obtaining "snapshots" of the solution to the primal and dual problems for all points in the sets $S_{N}^{\mathrm{pr}}$ and $S_{M}^{\mathrm{du}}$ respectively:

$$
W_{N}^{\mathrm{pr}}=\operatorname{span}\left\{\zeta_{i} \equiv u^{q}\left(\tilde{\mu}_{i}^{\mathrm{pr}}\right), i=1, \ldots, N, \tilde{\mu}_{i}^{\mathrm{pr}} \in S_{N}^{\mathrm{pr}}\right\}, W_{M}^{\mathrm{du}}=\operatorname{span}\left\{\xi_{i} \equiv \psi^{q}\left(\tilde{\mu}_{i}^{\mathrm{du}}\right), i=1, \ldots, M, \tilde{\mu}_{i}^{\mathrm{du}} \in S_{M}^{\mathrm{du}}\right\} ;
$$

recall that, from the definition of $S_{N}^{\mathrm{pr}}$ and $S_{N}^{\mathrm{du}}$, the "snapshots" are for different points in parameter space and time. The reduced-basis approximation to $u^{q}(t ; \mu)$ can be obtained by a standard Galerkin projection: for a given $\mu \in \mathcal{D}$, find $u_{N}^{q}(\cdot ; \mu) \in V^{q}\left(\mathcal{I} ; W_{N}^{\mathrm{pr}}\right)$, such that

$$
\int_{I}\left(\partial_{t} u_{N}^{q}(t ; \mu), v(t)\right) d t+\int_{I} a\left(u_{N}^{q}(t ; \mu), v(t) ; \mu\right) d t+\left(\left[u_{N}^{q}(\cdot ; \mu)\right]_{l-1}, v\left(t_{l-1}^{+}\right)\right)=\int_{I}(f(t ; \mu), v(t)) d t,
$$

$\forall v \in V^{q}\left(\mathcal{I} ; W_{N}^{\mathrm{pr}}\right)$ with $\left[u_{N}^{q}(\cdot ; \mu)\right]_{0}=u_{N}^{q}\left(0^{+} ; \mu\right)-u_{0}(\mu)$; similarly, we define the dual problem and obtain $\psi_{M}^{q}(\cdot ; \mu) \in V^{q}\left(\mathcal{I} ; W_{M}^{\mathrm{du}}\right)$. The primal and dual residuals are defined as: $\mathcal{R}^{\operatorname{pr} q}(v ; \mu)=\sum_{l \in \mathcal{L}} \mathcal{R}_{l}^{\operatorname{pr} q}(v ; \mu)$ with $\mathcal{R}_{l}^{\mathrm{pr} q}(v ; \mu)$ the residual for the primal problem in the time interval $I_{l}$ :

$$
\begin{aligned}
\mathcal{R}_{l}^{\operatorname{pr} q}(v ; \mu) & =\int_{I_{l}}(f(t ; \mu), v(t)) d t-\int_{I_{l}}\left(\partial_{t} u_{N}^{q}(t ; \mu), v(t)\right) d t-\int_{I_{l}} a\left(u_{N}^{q}(t ; \mu), v(t) ; \mu\right) d t-\left(\left[u_{N}^{q}(\cdot ; \mu)\right]_{l-1}, v\left(t_{l-1}^{+}\right)\right) \\
& =\int_{I_{l}}\left(\partial_{t} e^{\operatorname{pr} q}(t ; \mu), v(t)\right) d t+\int_{I_{l}} a\left(e^{\operatorname{pr} q}(t ; \mu), v(t) ; \mu\right) d t+\left(\left[e^{\operatorname{pr} q}(\cdot ; \mu)\right]_{l-1}, v\left(t_{l-1}^{+}\right)\right) ;
\end{aligned}
$$

where $e^{\operatorname{pr} q}(t ; \mu) \equiv u^{q}(t ; \mu)-u_{N}^{q}(t ; \mu)$, the error in the primal variable. The residual for the dual problem $\mathcal{R}_{l}^{\mathrm{du} q}(v ; \mu)$ is defined as:

$$
\begin{aligned}
\mathcal{R}_{l}^{\mathrm{du} q}(v ; \mu) & =-\int_{I_{l}}\left(\ell^{O}(t), v(t)\right) d t+\int_{I_{l}}\left(\partial_{t} \psi_{M}^{q}(t ; \mu), v(t)\right) d t-\int_{i l} a\left(v(t), \psi_{M}^{q}(t ; \mu) ; \mu\right) d t+\left(\left[\psi_{M}^{q}(\cdot ; \mu)\right]_{l}, v\left(t_{l}^{-}\right)\right) \\
& =-\int_{I_{l}}\left(\partial_{t} e^{\mathrm{du} q}(t ; \mu), v(t)\right) d t+\int_{I_{l}} a\left(v(t), e^{\mathrm{du} q}(t ; \mu) ; \mu\right) d t-\left(\left[e^{\mathrm{du} q}(\cdot ; \mu)\right]_{l}, v\left(t_{l}^{-}\right)\right) ;
\end{aligned}
$$


from (25) and defining $e^{\mathrm{du} q}(t ; \mu)=\psi^{q}(t ; \mu)-\psi_{M}^{q}(t ; \mu)$, the error in the dual variable. An approximation to the output of interest $s_{N}^{q}(\mu)$ can then be obtained from:

$$
s_{N}^{q}(\mu)=\sum_{l \in \mathcal{L}}\left[\int_{I_{l}}\left(\ell^{O}(t), u_{N}^{q}(t ; \mu)\right) d t-\mathcal{R}_{l}^{\operatorname{pr} q}\left(\psi_{M}^{q}(\cdot ; \mu) ; \mu\right)\right]+\left(g^{O}, u_{N}^{q}\left(T^{-} ; \mu\right)\right) .
$$

Turning now to the a posteriori error estimator, we compute "representations" of the error $\hat{e}^{\operatorname{pr} q}(\cdot ; \mu) \in$ $V^{q}(\mathcal{I} ; V)$ with $\left.\hat{e}_{l}^{\operatorname{pr} q}(\cdot ; \mu) \equiv \hat{e}^{\operatorname{pr} q}(\cdot ; \mu)\right|_{I_{l}}$, and $\hat{e}^{\mathrm{du} q}(\cdot ; \mu) \in V^{q}(\mathcal{I} ; V)$ with $\left.\hat{e}_{l}^{\text {du } q}(\cdot ; \mu) \equiv \hat{e}^{\text {du } q}(\cdot ; \mu)\right|_{I_{l}}$ such that:

$$
\begin{aligned}
g(\mu) \int_{I_{l}} \hat{a}\left(\hat{e}_{l}^{\operatorname{pr} q}(t ; \mu), v(t)\right) d t & =\mathcal{R}_{l}^{\operatorname{pr} q}(v ; \mu), \forall v \in \mathbb{P}^{q}\left(I_{l} ; V\right), \forall I_{l} \in \mathcal{I} \text { and } \\
g(\mu) \int_{I_{l}} \hat{a}\left(\hat{e}_{l}^{\operatorname{du} q}(t ; \mu), v(t)\right) d t & =\mathcal{R}_{l}^{\operatorname{du} q}(v ; \mu), \forall v \in \mathbb{P}^{q}\left(I_{l} ; V\right), \forall I_{l} \in \mathcal{I} .
\end{aligned}
$$

For the error estimator we first calculate the output approximation, $s_{B}^{q}(\mu)$ :

$$
s_{B}^{q}(\mu)=s_{N}^{q}(\mu)-\frac{g(\mu)}{2} \sum_{l \in \mathcal{L}} \int_{I_{l}} \hat{a}\left(\hat{e}_{l}^{\mathrm{pr} q}(t ; \mu), \hat{e}_{l}^{\mathrm{du} q}(t ; \mu)\right) d t
$$

and the bound gap $\Delta^{q}(\mu)$ is defined as:

$$
\Delta^{q}(\mu)=\frac{g(\mu)}{2}\left[\sum_{l \in \mathcal{L}} \int_{I_{l}} \hat{a}\left(\hat{e}_{l}^{\operatorname{pr} q}(t ; \mu), \hat{e}_{l}^{\operatorname{pr} q}(t ; \mu)\right) d t\right]^{\frac{1}{2}}\left[\sum_{l \in \mathcal{L}} \int_{I_{l}} \hat{a}\left(\hat{e}_{l}^{\mathrm{du} q}(t ; \mu), \hat{e}_{l}^{\mathrm{du} q}(t ; \mu)\right) d t\right]^{\frac{1}{2}} .
$$

Finally, as before, symmetric upper and lower output estimators can be calculated from $s^{ \pm q}(\mu)=s_{B}^{q}(\mu) \pm$ $\Delta^{q}(\mu)$. We can then prove the following:

Proposition 2. Let $s^{q}(\mu)$ be the exact value of the output for the semi-discrete problem, defined in (26). If we define $s_{B}^{q}(\mu)$ and $\Delta^{q}(\mu)$ as in (28) and (29), respectively, then $s^{ \pm q}(\mu)=s_{B}^{q}(\mu) \pm \Delta^{q}(\mu)$ are upper and lower bounds to the true output:

$$
s^{-q}(\mu) \leq s^{q}(\mu) \leq s^{+q}(\mu), \forall \mu \in \mathcal{D} .
$$

Proof. We first obtain some results for $\sum_{l \in \mathcal{L}} \mathcal{R}_{l}^{\operatorname{pr} q}\left(e^{\mathrm{pr} q} ; \mu\right)$ and $\sum_{l \in \mathcal{L}} \mathcal{R}_{l}^{\mathrm{du} q}\left(e^{\mathrm{pr} q} ; \mu\right)$ that will be required in the following. First we look in the error for the output, which from the definition of $s^{q}(\mu)(26)$ and $s_{N}^{q}(\mu)$ (27) becomes:

$$
s^{q}(\mu)-s_{N}^{q}(\mu)=\sum_{l \in \mathcal{L}}\left[-\int_{I_{l}}\left(\partial_{t} e^{\operatorname{pr} q}, \psi^{q}\right) d t-\int_{I_{l}} a\left(e^{\operatorname{pr} q}, \psi^{q} ; \mu\right) d t+\mathcal{R}_{l}^{\operatorname{pr} q}\left(\psi_{M}^{q}(\cdot ; \mu) ; \mu\right)\right]+I_{1} ;
$$

using (25) and integration by parts. The additional terms $I_{1}$ can be simplified, as follows:

$$
\begin{aligned}
I_{1} & =\sum_{l \in \mathcal{L}}\left[\left(\left[\psi^{q}\right]_{l}, e^{\operatorname{pr} q}\left(t_{l}^{-}\right)\right)+\left(\psi^{q}\left(t_{l}^{-}\right), e^{\operatorname{pr} q}\left(t_{l}^{-}\right)\right)-\left(\psi^{q}\left(t_{l-1}^{+}\right), e^{\operatorname{pr} q}\left(t_{l-1}^{+}\right)\right)\right]+\left(g^{O}(\mu), e^{\operatorname{pr} q}\left(T^{-}\right)\right) \\
& =\sum_{l \in \mathcal{L}}\left[\left(\psi^{q}\left(t_{l}^{+}\right), e^{\operatorname{pr} q}\left(t_{l}^{-}\right)\right)-\left(\psi^{q}\left(t_{l-1}^{+}\right), e^{\operatorname{pr} q}\left(t_{l-1}^{-}\right)\right)-\left(\psi^{q}\left(t_{l-1}^{+}\right),\left[e^{\operatorname{pr} q}\right]_{l-1}\right)\right]+\left(g^{O}(\mu), e^{\operatorname{pr} q}\left(T^{-}\right)\right) \\
& =\left(g^{O}(\mu), e^{\operatorname{pr} q}\left(T^{-}\right)\right)+(\underbrace{\psi^{q}\left(T^{+}\right)}_{-g^{O}(\mu)}, e^{\operatorname{pr} q}\left(T^{-}\right))-(\psi^{q}\left(0^{+}\right), \underbrace{e^{\operatorname{pr} q}\left(0^{-}\right)}_{0})-\sum_{l \in \mathcal{L}}\left(\left[e^{\operatorname{pr} q}\right]_{l-1}, \psi^{q}\left(t_{l-1}^{+}\right)\right) ;
\end{aligned}
$$


using in the second line the definition of the jump operator $e^{\operatorname{pr} q}\left(t_{l-1}^{+}\right)=\left[e^{\operatorname{pr} q}\right]_{l-1}+e^{\operatorname{pr} q}\left(t_{l-1}^{-}\right)$; and in the last line, $\psi^{q}\left(T^{+} ; \mu\right)=-g^{O}$ from the definition of the dual problem, and $e^{\operatorname{pr} q}\left(0^{-} ; \mu\right)=0$. Therefore,

$$
\begin{aligned}
& s^{q}(\mu)-s_{N}^{q}(\mu)= \\
& \quad=\sum_{l \in \mathcal{L}}\left[-\int_{I_{l}}\left(\partial_{t} e^{\operatorname{pr} q}, \psi^{q}\right) d t-\int_{I_{l}} a\left(e^{\operatorname{pr} q}, \psi^{q} ; \mu\right) d t-\left(\left[e^{\operatorname{pr} q}\right]_{l-1}, \psi^{q}\left(t_{l-1}^{+}\right)\right)+\mathcal{R}_{l}^{\operatorname{pr} q}\left(\psi_{M}^{q}(\cdot ; \mu) ; \mu\right) ;\right] \\
& =-\sum_{l \in \mathcal{L}} \mathcal{R}_{l}^{\operatorname{pr} q}\left(e^{\operatorname{du} q} ; \mu\right) .
\end{aligned}
$$

But $\sum_{l \in \mathcal{L}} \mathcal{R}_{l}^{\operatorname{pr} q}\left(e^{\mathrm{du} q} ; \mu\right)=\sum_{l \in \mathcal{L}} \mathcal{R}_{l}^{\mathrm{du} q}\left(e^{\mathrm{pr} q} ; \mu\right)$, since

$$
\begin{aligned}
\sum_{l \in \mathcal{L}} \mathcal{R}_{l}^{\operatorname{pr} q}\left(e^{\mathrm{du} q} ; \mu\right) & =\sum_{l \in \mathcal{L}}\left[\int_{I_{l}}\left(\partial_{t} e^{\mathrm{pr} q}, e^{\mathrm{du} q}\right) d t+\int_{I_{l}} a\left(e^{\mathrm{pr} q}, e^{\mathrm{du} q} ; \mu\right) d t+\left(\left[e^{\mathrm{pr} q}\right]_{l-1}, e^{\mathrm{du} q}\left(t_{l-1}^{+}\right)\right)\right] \\
& =\sum_{l \in \mathcal{L}}\left[-\int_{I_{l}}\left(\partial_{t} e^{\mathrm{du} q}, e^{\operatorname{pr} q}\right) d t+\int_{I_{l}} a\left(e^{\operatorname{pr} q}, e^{\mathrm{du} q} ; \mu\right) d t\right]+I_{2} \\
& =\sum_{l \in \mathcal{L}}\left[-\int_{I_{l}}\left(\partial_{t} e^{\mathrm{du} q}, e^{\operatorname{pr} q}\right) d t+\int_{I_{l}} a\left(e^{\operatorname{pr} q}, e^{\mathrm{du} q} ; \mu\right) d t-\left(\left[e^{\mathrm{du} q}\right]_{l}, e^{\mathrm{pr} q}\left(t_{l}^{-}\right)\right)\right] \\
& =\sum_{l \in \mathcal{L}} \mathcal{R}_{l}^{\mathrm{du} q}\left(e^{\operatorname{pr} q} ; \mu\right) ;
\end{aligned}
$$

from integration by parts and the definitions of the primal and dual residuals. The additional terms $I_{2}$ are calculated below:

$$
\begin{aligned}
I_{2} & =\sum_{l \in \mathcal{L}}\left[\left(e^{\operatorname{pr} q}\left(t_{l}^{-}\right), e^{\mathrm{du} q}\left(t_{l}^{-}\right)\right)-\left(e^{\operatorname{pr} q}\left(t_{l-1}^{+}\right), e^{\mathrm{du} q}\left(t_{l-1}^{+}\right)\right)+\left(e^{\operatorname{pr} q}\left(t_{l-1}^{+}\right)-e^{\operatorname{pr} q}\left(t_{l-1}^{-}\right), e^{\mathrm{du} q}\left(t_{l-1}^{+}\right)\right)\right] \\
& =\sum_{l \in \mathcal{L}}\left[-\left(e^{\operatorname{pr} q}\left(t_{l}^{-}\right),\left[e^{\mathrm{du} q}\right]_{l}\right)+\left(e^{\operatorname{pr} q}\left(t_{l}^{-}\right), e^{\mathrm{du} q}\left(t_{l}^{+}\right)\right)-\left(e^{\operatorname{pr} q}\left(t_{l-1}^{-}\right), e^{\mathrm{du} q}\left(t_{l-1}^{+}\right)\right)\right] \\
& =-\sum_{l \in \mathcal{L}}\left(\left[e^{\mathrm{du} q}\right]_{l}, e^{\operatorname{pr} q}\left(t_{l}^{-}\right)\right) ;
\end{aligned}
$$

again the definition of the jump operator has been used, and $e^{\operatorname{pr} q}\left(0^{-}\right)=e^{\mathrm{du} q}\left(T^{+}\right)=0$. Combining (30) and (31) we obtain:

$$
-\sum_{l \in \mathcal{L}} \mathcal{R}_{l}^{\mathrm{du} q}\left(e^{\mathrm{pr} q} ; \mu\right)=\left(s^{q}(\mu)-s_{N}^{q}(\mu)\right) .
$$

Turning now to $\sum_{l \in \mathcal{L}} \mathcal{R}_{l}^{\operatorname{pr} q}\left(e^{\operatorname{pr} q} ; \mu\right)$, we first compute $I_{3}$

$$
\begin{aligned}
& I_{3}=\sum_{l \in \mathcal{L}}\left[\frac{1}{2}\left(e^{\operatorname{pr} q}\left(t_{l}^{-}\right), e^{\operatorname{pr} q}\left(t_{l}^{-}\right)\right)-\frac{1}{2}\left(e^{\operatorname{pr} q}\left(t_{l-1}^{+}\right), e^{\operatorname{pr} q}\left(t_{l-1}^{+}\right)\right)+\left(e^{\operatorname{pr} q}\left(t_{l-1}^{+}\right)-e^{\operatorname{pr} q}\left(t_{l-1}^{-}\right), e^{\operatorname{pr} q}\left(t_{l-1}^{+}\right)\right)\right] \\
& =\sum_{l \in \mathcal{L}} \frac{1}{2}\left\|\left[e^{\operatorname{pr} q}\left(t_{l-1}\right)\right]\right\|_{L^{2}(\Omega)}^{2}+\frac{1}{2}\left(e^{\operatorname{pr} q}\left(T^{-}\right), e^{\operatorname{pr} q}\left(T^{-}\right)\right)-\frac{1}{2}(\underbrace{e^{\operatorname{pr} q}\left(0^{-}\right)}_{0}, e^{\operatorname{pr} q}\left(0^{-}\right)) ;
\end{aligned}
$$

as before we used here the definition of the jump operator and simple algebraic manipulations. But then,

$$
\begin{aligned}
\sum_{l \in \mathcal{L}} \mathcal{R}_{l}^{\operatorname{pr} q}\left(e^{\operatorname{pr} q} ; \mu\right) & =\sum_{l \in \mathcal{L}}\left[\int_{I_{l}}\left(\partial_{t} e^{\operatorname{pr} q}, e^{\operatorname{pr} q}\right) d t+\int_{I_{l}} a\left(e^{\operatorname{pr} q}, e^{\operatorname{pr} q} ; \mu\right) d t+\left(\left[e^{\operatorname{pr} q}\right]_{l-1}, e^{\operatorname{pr} q}\left(t_{l-1}^{+}\right)\right)\right] \\
& =I_{3}+\sum_{l \in \mathcal{L}} \int_{I_{l}} a\left(e^{\operatorname{pr} q}, e^{\operatorname{pr} q} ; \mu\right) d t \geq g(\mu) \int_{I} \hat{a}\left(e^{\operatorname{pr} q}, e^{\operatorname{pr} q}\right) d t
\end{aligned}
$$


since $I_{3}$ is the sum of non-negative terms, and also using (14). We turn now to the proof of the bounding properties, and as before, for $\kappa>0$ we define $\hat{e}^{ \pm q}=\hat{e}^{\operatorname{pr} q} \mp \frac{1}{\kappa} \hat{e}^{\mathrm{du} q}$. From the coercivity of $\hat{a}$,

$$
\begin{gathered}
\kappa g(\mu) \int_{I} \hat{a}\left(e^{\operatorname{pr} q}-\frac{1}{2} \hat{e}^{ \pm q}, e^{\operatorname{pr} q}-\frac{1}{2} \hat{e}^{ \pm q}\right) d t \geq 0 \\
\kappa g(\mu) \int_{I} \hat{a}\left(e^{\operatorname{pr} q}, e^{\operatorname{pr} q}\right) d t+\frac{\kappa g(\mu)}{4} \int_{0}^{T} \hat{a}\left(\hat{e}^{ \pm q}, \hat{e}^{ \pm q}\right) d t-\kappa g(\mu) \int_{I} \hat{a}\left(\hat{e}^{ \pm q}, e^{\operatorname{pr} q}\right) d t \geq 0 .
\end{gathered}
$$

From the definition of $\hat{e}^{ \pm q}, \hat{e}^{\mathrm{pr} q}$, and $\hat{e}^{\mathrm{du} q}$ we have:

$$
g(\mu) \int_{I} \hat{a}\left(\hat{e}^{ \pm q}, e^{\operatorname{pr} q}\right) d t=\sum_{l \in \mathcal{L}} \mathcal{R}_{l}^{\operatorname{pr} q}\left(e^{\operatorname{pr} q} ; \mu\right) \mp \frac{1}{\kappa} \sum_{l \in \mathcal{L}} \mathcal{R}_{l}^{\mathrm{du} q}\left(e^{\operatorname{pr} q} ; \mu\right) .
$$

Using (33) to replace $\sum_{l \in \mathcal{L}} \mathcal{R}_{l}^{\operatorname{pr} q}\left(e^{\operatorname{pr} q} ; \mu\right)$, and (32) to replace $\sum_{l \in \mathcal{L}} \mathcal{R}_{l}^{\text {du } q}\left(e^{\operatorname{pr} q} ; \mu\right)$, we get

$$
-\kappa g(\mu) \int_{I} \hat{a}\left(\hat{e}^{ \pm q}, e^{\operatorname{pr} q}\right) d t \leq-\kappa g(\mu) \int_{I} \hat{a}\left(e^{\operatorname{pr} q}, e^{\operatorname{pr} q}\right) d t \mp\left(s^{q}(\mu)-s_{N}^{q}(\mu)\right) .
$$

Replacing now (35) in (34), we get

$$
\pm\left(s^{q}(\mu)-s_{N}^{q}(\mu)\right) \leq \frac{\kappa g(\mu)}{4} \int_{I} \hat{a}\left(\hat{e}^{ \pm q}, \hat{e}^{ \pm q}\right) d t .
$$

The rest of the proof follows similarly to that of the continuous case.

To measure the quality of the computed bounds, we define the a posteriori effectivity index $\eta(\mu)$, as the ratio of the computed error over the true error in the output prediction

$$
\eta(\mu)=\frac{\Delta^{q}(\mu)}{\left|s^{q}(\mu)-s_{B}^{q}(\mu)\right|} .
$$

According to the previous theorem, the prediction of the error in the output will overestimate the true error and therefore the effectivity will always be larger than one, $\eta(\mu) \geq 1, \forall \mu \in \mathcal{D}$. One needs to be careful with the definition of the effectivity above: for certain parameters, it might happen that the output prediction $s_{B}^{q}(\mu)$ is very close to the true error $s^{q}(\mu)$ (in the worst case, equal) ${ }^{1}$ and this implies large effectivities (in the worst case, infinite). This might not necessarily mean that the reduced-basis prediction is inaccurate: in these cases we need to look at the bound gap and its magnitude. When interpreting the effectivity it is best to compute the average value (or the median) of the effectivity for a large number of test points. Large effectivities (on the average) suggest that the computed error bound largely overestimates the true error and therefore the bounds obtained are not sharp. This implies that for a given accuracy, the error estimator suggests the use of a larger-than-required number of basis functions and this leads to an unnecessary increase in the computational cost. For efficiency, it is therefore desired that the effectivities will be as close to one as possible. The choice of $\hat{a}$ and $g(\mu)$ is critical to obtain good effectivities; for a discussion see [16]. For better effectivities, it is possible to choose different $\hat{a}$ and $g(\mu)$ which satisfy (14) only in subregions of the parameter domain. Also the more general bound conditioners, developed in [22] for elliptic coercive problems, can also be extended to the parabolic case.

\section{Results}

We consider the problem of designing the thermal fin of Figure 1 to cool (say) an electronic component at the fin base, $\Gamma_{\text {root }}$. The $i$ th "radiator" of the fin has thermal conductivity $k_{i}$ (normalized relative to the

\footnotetext{
${ }^{1}$ Indeed $s^{q}(\mu)$ is a real number and by chance $s_{B}^{q}(\mu)$ might be equal to $s^{q}(\mu)$ but this does not imply anything on the approximation of the function $u$.
} 
conductivity of the central post), and the fluid surrounding the fin is characterized by the heat convection coefficient expressed in non-dimensional form by a Biot number $B i$. The fin geometry is described by the radiator length $\beta$ and the thickness $\alpha$, both non-dimensionalized with respect to the width of the fin base. We thus have $P=7$, with a typical point $\mu \in \mathcal{D} \subset \mathbb{R}^{7}$ given by $\mu=\left\{k_{1}, k_{2}, k_{3}, k_{4}, \mathrm{Bi}, \alpha, \beta\right\}$.

Initially, the non-dimensional temperature is $u_{0}(\mu)=$ 0 . A uniform heat flux is applied at the root of the fin at $t=0$ and remains on until the final time $t=T \equiv 3$. The temperature increases until it reaches the final value $u\left(T^{-} ; \mu\right)$. On the original domain the bilinear form is given by, $\tilde{a}(w, v ; \mu)=\int_{\tilde{\Omega}_{0}} \nabla w \cdot \nabla v+\sum_{i=1}^{4} k_{i} \int_{\tilde{\Omega}_{i}} \nabla w$. $\nabla v+\operatorname{Bi} \int_{\partial \tilde{\Omega} \backslash \Gamma_{\text {root }}} w v$; with $\tilde{\Omega}_{0}$ the fin central-post domain, and $\tilde{\Omega}_{i}$ the $i$ th radiator domain. We then map the original domain $\tilde{\Omega}$ to a reference geometry $\Omega$, shown by solid lines in Figure 1. The original bilinear form $\tilde{a}(w, v ; \mu)$ is replaced by $a(w, v ; \mu)$ defined in the fixed domain $\Omega$ - the variable geometry appears as domain-dependent effective orthotropic conductivities and Bi numbers. Similarly, the $L^{2}$-inner product $(w, v)_{L^{2}(\tilde{\Omega})}$ is replaced by $(w, v)_{L^{2}(\Omega)} \equiv b(w, v ; \mu)$, defined on the fixed domain the variable geometry also makes the $L^{2}$-inner product

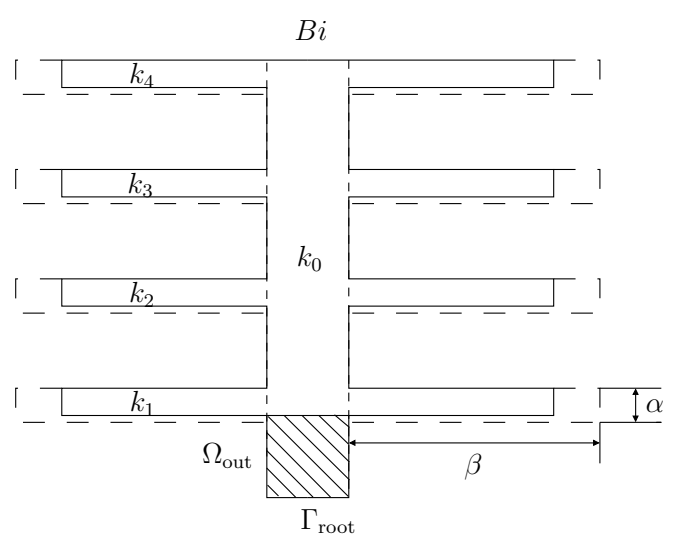

Figure 1: Two-dimensional thermal fin parameter-dependent. We consider two outputs: the first, is the mean temperature of the base $\Gamma_{\text {root }}$ averaged over the time interval $(0, T)$ :

$$
s^{1}(\mu) \equiv s^{1}(u(\cdot ; \mu))=\frac{1}{T} \int_{I} \int_{\Gamma_{\text {root }}} u(t ; \mu) d S d t
$$

the second, is the mean temperature in the shaded region $\Omega_{\text {out }}$ (with area $A_{\Omega_{\text {out }}}$ ) at the final time $t=T$ :

$$
s^{2}(\mu) \equiv s^{2}(u(t ; \mu))=\frac{1}{A_{\Omega_{\text {out }}}} \int_{\Omega_{\text {out }}} u\left(T^{-} ; \mu\right) .
$$

Both outputs are, to a certain extent, indicators of the cooling performance of the fin.

Taking advantage of the natural domain decomposition afforded by our mapping, it is not difficult to cast the problem such that the affine decomposition assumption is verified:

$$
\begin{aligned}
& a(w, v ; \mu)=\sum_{q=1}^{Q_{a}} \sigma_{a}^{q}(\mu) a^{q}(w, v), \forall w, v \in V^{2}, \text { and } \\
& b(w, v ; \mu)=\sum_{q=1}^{Q_{b}} \sigma_{b}^{q}(\mu) b^{q}(w, v), \forall w, v \in L^{2}(\Omega)
\end{aligned}
$$

with $Q_{a}=16$ and $Q_{b}=3$. Choosing

$$
\hat{a}(w, v)=\sum_{q=1}^{Q_{a}} a^{q}(w, v)=\int_{\Omega} \nabla w \cdot \nabla v+\int_{\partial \Omega \backslash \Gamma_{\text {root }}} w v,
$$

and $g(\mu)=\min _{q \in\left\{1, \ldots, Q_{a}\right\}} \sigma_{a}^{q}(\mu)$ (the $\sigma_{a}^{q}(\mu)$ are all bounded below by a positive constant), we are able to verify (14). Thus all the requirements are honored, and the bound method can be applied.

We choose the total (non-dimensional) height of the thermal fin $\hat{H}=4$, and the length and height of the radiators $\hat{\alpha}=2.5$ and $\hat{\beta}=0.25$ respectively; the reference geometry $\hat{\Omega}$ is thus completely defined. To obtain the "exact" solution: first, for the spatial discretization, we introduce a very fine triangulation $\mathcal{T}_{h}$ and define 


\begin{tabular}{|c|c|c|}
\hline$N=M$ & $\frac{\left|s_{N}^{1}\left(\mu_{t}\right)-s^{1}\left(\mu_{t}\right)\right|}{s^{1}\left(\mu_{t}\right)}$ & $\frac{\left|s_{N}^{2}\left(\mu_{t}\right)-s^{2}\left(\mu_{t}\right)\right|}{s^{2}\left(\mu_{t}\right)}$ \\
\hline 8 & $1.22 e-01$ & $2.03 e-01$ \\
20 & $3.15 e-03$ & $6.41 e-03$ \\
40 & $1.18 e-04$ & $4.61 e-04$ \\
60 & $3.91 e-05$ & $3.02 e-05$ \\
80 & $1.16 e-06$ & $2.56 e-06$ \\
100 & $7.42 e-07$ & $1.22 e-06$ \\
120 & $1.74 e-08$ & $2.46 e-07$ \\
\hline
\end{tabular}

Table 1: Relative error by the reduced-basis prediction of the outputs of interest for different values of $N=M$.

the finite-element space $V \approx V_{h}=\left\{v \in H^{1}(\Omega)|v|_{T_{h}} \in \mathbb{P}^{1}, \forall T_{h} \in \mathcal{T}_{h}\right\}$ with piecewise linear polynomials over each of the elements $T_{h}$; and second, for the temporal discretization, the discontinuous Galerkin method is used with $q=0$ and the time interval $\bar{I}=[0,3]$ is partitioned into $L=30$ intervals of uniform length $\Delta \tau^{l} \equiv \Delta \tau=0.1, \forall l \in \mathcal{L}$. (The same parameters are used for the reduced-basis problems.)

Next in the definition of our problem, is the specification of the ranges for each of the input parameters. We choose a parameter space as follows: $\mathcal{D}=[0.01,100.0]^{4} \times[0.001,10.0] \times[0.2,0.6] \times[2.3,2.8]$, that is $0.01 \leq k_{1,2,3,4} \leq 100.0,0.001 \leq \mathrm{Bi} \leq 10.0,0.2 \leq \alpha \leq 0.6$ and $2.3 \leq \beta \leq 2.8$. Points in this parameter space - for example, for the construction of the sample sets $S_{N}^{\mathrm{pr}}$ and $S_{M}^{\mathrm{du}}$ - are obtained by sampling "log-randomly" (see Section 3). A point $\mu \in \mathcal{D}$, describes a particular configuration. For example, $\mu_{t}=$ $\{0.4,0.6,0.8,1.2,0.1,0.3,2.8\}$ represents a thermal fin with $k_{1}=0.4, k_{2}=0.6, k_{3}=0.8, k_{4}=1.2, \mathrm{Bi}=0.1$, $\alpha=0.3$, and $\beta=2.8$; this particular configuration will be used as a test point $\mu_{t}$ in the following numerical experiments.

For the construction of the primal reduced-basis space we sample $\mathcal{D}$ and obtain a number of points $\mu_{i}^{\mathrm{pr}}$. For each of these points the primal problem is solved and the reduced-basis vectors are obtained by taking "snapshots" of the solution at different times. The sampling times or the number of snapshots can vary arbitrarily from one configuration to the next; in the following, for each configuration, four "snapshots" were obtained at $t=1 \Delta \tau, 10 \Delta \tau, 20 \Delta \tau$, and $30 \Delta \tau$. For example if $N=20$, five different configurations were considered, each giving four basis vectors for the construction of the reduced-basis space. For the dual reduced-basis space the same procedure is followed, solving the dual problem for a different set of parameter points and taking "snapshots" of the solution at $t=29 \Delta \tau, 20 \Delta \tau, 10 \Delta \tau$, and $0 \Delta \tau$.

As a first test, we study the convergence of the reduced-basis solution to the exact one. For this, we sample $\log$-randomly the parameter space $\mathcal{D}$ and construct reduced-basis spaces of increasing dimension $N=M$. Using these spaces we compute, for the test point $\mu_{t}$, the reduced-basis solution and the two outputs of interest. In Table 1, the error in the prediction of the adjoint-corrected output relative to the exact value, is shown for increasing values of $N$. We can see that, for both outputs, the output prediction converges very fast to the exact value, albeit at a different rate for each output. If, for example, a $1 \%$ accuracy is required - which is sufficient in many engineering applications, - then only $N=20$ basis functions would be sufficient. This implies that the incremental cost for each new output evaluation is very small; depending on the dimension of the space $V_{h}$, the computational savings can be of several orders of magnitude. For sufficiently large values of $N, M$ the vectors that comprise the reduced basis spaces are closely related and this leads to ill-conditioning problems. Indeed in our case increasing $N, M$ above 120, ill-conditioning leads first to deterioration of the convergence rate and eventually to incorrect results. The issue of ill-conditioning in the reduced-basis context is very important, but an analysis will not be further pursued; first, because we are usually interested in the pre-asymptotic region (small values of $N$ ); and second, because even for the conservative triangulation used here, the discretization error is of the same order of magnitude as the reduced-basis error when $N=M=80$ - using higher values for $N$ is not relevant except, maybe, for testing the convergence rate. 


\begin{tabular}{|c|c|c|c|c|c|}
\hline$N$ & 20 & 40 & 60 & 80 & 100 \\
$M$ & 100 & 80 & 60 & 40 & 20 \\
\hline$\Delta^{1}\left(\mu_{t}\right)$ & $1.10 e-03$ & $7.62 e-04$ & $8.39 e-04$ & $1.22 e-03$ & $9.98 e-04$ \\
$\Delta^{2}\left(\mu_{t}\right)$ & $2.78 e-03$ & $2.10 e-03$ & $2.33 e-03$ & $3.25 e-03$ & $3.47 e-03$ \\
$\eta^{1}\left(\mu_{t}\right)$ & 34.2 & 53.0 & 13.7 & 41.7 & 106.1 \\
$\eta^{2}\left(\mu_{t}\right)$ & 184.8 & 22.3 & 16.5 & 33.1 & 88.2 \\
\hline
\end{tabular}

Table 2: Bound gap and effectivities for the two outputs of interest, for different choices of $N=\operatorname{dim} W_{N}^{\mathrm{pr}}$ and $M=\operatorname{dim} W_{M}^{\mathrm{du}}(\mathrm{N}+\mathrm{M}=120)$.

The choice of the sample set $S_{N}^{\mathrm{pr}}$, is critical for the approximation properties of $W_{N}^{\mathrm{pr}}$. For the same $N$, different choices for $S_{N}^{\mathrm{pr}}$ can give different reduced-basis spaces and consequently different output approximations; relatedly the approximation error can vary significantly for different test points $\mu \in \mathcal{D}$. Moreover, even for the same sample set $S_{N}^{\mathrm{pr}}$, the error for different outputs can be quite different. For example, in Table 1 , for $N=40$ and the particular point $\mu_{t}$, the error in the prediction of the second output is four times larger compared to the error in the first output. Ascertaining the accuracy of our predictions without, of course, computing the exact solution, is therefore critical for the successful application of the reduced-basis method; the importance of efficient and reliable methods to a posteriori estimate the error in our predictions should be clear.

We turn now to the a posteriori error estimator procedure and investigate its behavior in the context of the model problem. To calculate the bounds, we need to solve using the reduced-basis method both the primal problem of dimension $N$, and the dual problem of dimension $M$. These dimensions determine the accuracy of the approximation to each of the problems and can, in principle, be chosen independently. To understand how this choice affects the accuracy of the predictions, we fix the total dimension $N+M=120$ and choose different combinations for $N$ and $M$. In Table 2, for the particular point $\mu_{t}$ and the two outputs of interest, the bound gap $\Delta\left(\mu_{t}\right)$ and the effectivity $\eta\left(\mu_{t}\right)$ are presented for different choices of $N$ and $M$.

To understand the behavior the bound gap, recall that it is defined (29) as the product of norms of representations to the primal $\hat{e}^{\mathrm{pr}}$ and dual errors $\hat{e}^{\mathrm{du}}$ - which are directly related to the true errors. As $N$ increases the error in the primal solution becomes smaller, while at the same time, $M$ is decreasing and the error in the dual solution becomes larger. Therefore, as we can verify from Table 2, the bound gap does not change appreciably for the different $N$ and $M$. The small variations can also be attributed to the different selection of basis functions in the formation of the reduced-basis spaces. On the other hand, the dual correction term in the output approximation (27), ensures that the output will be more accurate when either $N$ or $M$ are large. In these cases the error in the output is small and given that the bound gap does not change significantly, justifies the higher effectivities. The discussion above suggests that, for a given accuracy — as dictated by the bound gap $\Delta(\mu)$, — we can choose $N$ or $M$ arbitrarily, such that the total number of basis functions is constant. On one side, we have the case $M=0(N=0)$, which corresponds to a pure primal (dual) problem; on the other, we can have a mixed approach with $N=M$. The computational cost for the second case is roughly two times smaller in the off-line stage and four times smaller in the on-line. The use of both the primal and the adjoint problems is thus dictated by computational efficiency considerations.

As a final test, we choose $N=M$ and for the test point $\mu_{t}$, we vary the dimension of the reduced-basis spaces. The behavior of the bound gap as a function of $N=M$ is shown in Figure 2, and of the effectivity in Figure 3. Despite the relatively high dimension of the parameter space, we observe the good accuracy and rapid convergence of the bound gap. Also, given that the effectivity remains bounded for all values of $N$, we conclude that the bound gap converges at the same rate as the true error in the output. This suggests that instead of using the high-dimensional model to evaluate outputs for different parameter points, we can replace it with a reduced-basis model. Due to the rapid convergence only a few basis functions are required and therefore we can obtain high efficiency. In addition, we recover certainty as the error bounds validate the accuracy of the reduced-basis predictions. In terms of computational effort, the off-line stage 


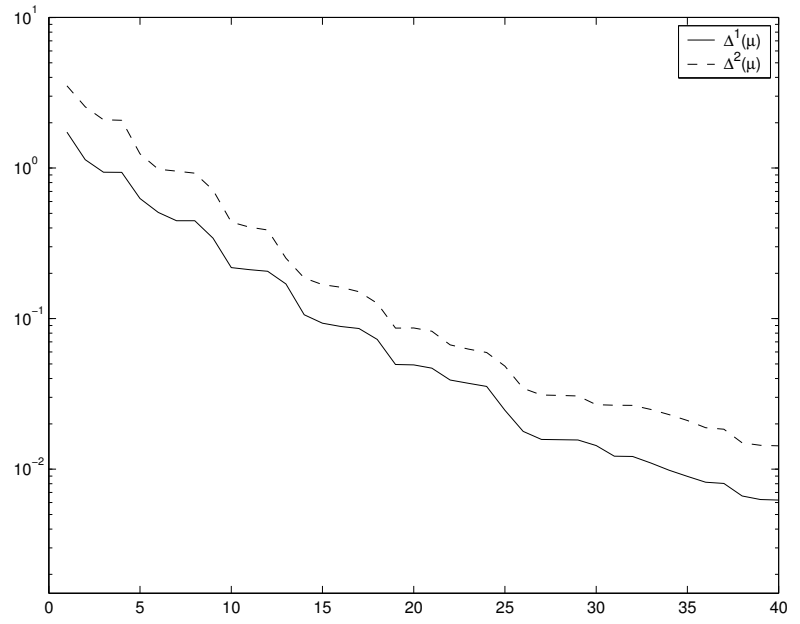

Figure 2: Convergence of the bound gap as a function of $\mathrm{N}(=\mathrm{M})$, for the point $\mu_{t}$.

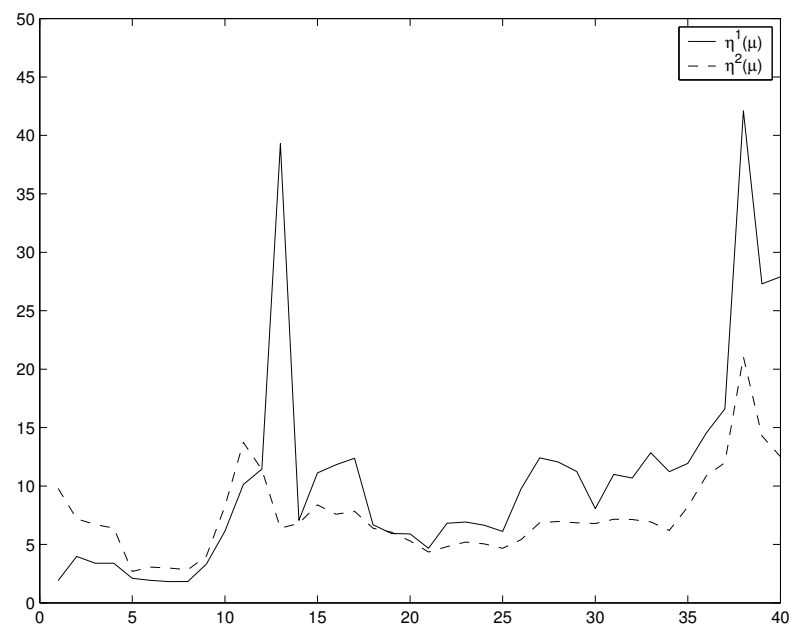

Figure 3: Effectivity as a function of $\mathrm{N}(=\mathrm{M})$ for the point $\mu_{t}$. Note the presence of two outliers for $N=13,38$ that do not correspond to accidents on the convergence of the bound gap but better approximation for $s_{B}^{q}\left(\mu_{t}\right)$ from which we shouldn't conclude that it is necessary to change $N$.

requires, typically, a few hundred solutions of the continuous problem - depending on the number of basis functions and the parametric complexity of the bilinear forms. But then the on-line cost, for each new configuration $\mu \in \mathcal{D}$ is typically more than a hundred or a thousand times smaller - depending on the dimension of $V_{h}$. The computational advantages in the limit of many evaluations, should be obvious. More realistic applications, as well as integration of these components in an optimization or design framework will be addressed in a future paper.

Acknowledgements Many of the ideas in this paper originate in our joint work with Professor Anthony T. Patera of Massachusetts Institute of Technology. We would also like to thank him for helpful comments on reduced-basis methods and a posteriori error estimation in general. This work was performed while D.R. was at M.I.T and was supported by the Singapore-MIT Alliance. Part of this work was done at the University of Paris VI, while D.R. was partially supported by a Chateaubriand Fellowship.

\section{References}

[1] B. O. Almroth, P. Stern, and F. A. Brogan. Automatic choice of global shape functions in structural analysis. AIAA Journal, 16:525-528, May 1978.

[2] E. Balmes. Parametric families of reduced finite-element models. theory and applications. Mechanical Systems and Signal Processing, 10(4):381-394, 1996.

[3] M. Barrault, Y. Maday, N.C. Nguyen, and A.T. Patera. An 'empirical interpolation' method: application to efficient reuced-basis discretization of partial differential equations. C. R. Acad. Sci., Paris, Ser. I, 2004. To appear.

[4] P. Feldmann and R.W. Freund. Efficient linear circuit analysis by pade-approximation via the lanczos process. IEEE Transactions on Computer-Aided Design of Integrated Circuits and Systems, 14(5):639649, May 1995. 
[5] M. Grepl. PhD thesis, Massachusetts Institute of Technology, 2004. In progress.

[6] Pierre Jamet. Galerkin-type approximations which are discontinuous in time for parabolic equations in a variable domain. SIAM Journal on Numerical Analysis, 15(5):912-928, Oct. 1978.

[7] K. Karhunen. Zur spektraltheorie stochastischer prozesse. Annales Academiae Scientiarum Fennicae, $37,1946$.

[8] P. Krysl, S. Lall, and J.E. Marsden. Dimensional model reduction in non-linear finite element dynamics of solids and structures. International Journal for Numerical Methods in Engineering, 51:479-504, 2001.

[9] J.L. Lions and E. Magenes. Non-Homogenous Boundary Value Problems and Applications. SpringerVerlag, 1972.

[10] MM. Loeve. Probablity Theory. Van Nostrand, 1955.

[11] L. Machiels, Y. Maday, I. B. Oliveira, A.T. Patera, and D.V. Rovas. Output bounds for reduced-basis approximations of symmetric positive definite eigenvalue problems. C. R. Acad. Sci. Paris, Série I, 331(2):153-158, July 2000.

[12] Y. Maday, L. Machiels, A. T. Patera, and D. V. Rovas. Blackbox reduced-basis output bound methods for shape optimization. In Proceedings $12^{\text {th }}$ International Domain Decomposition Conference, pages 429-436, Chiba, Japan, 2000.

[13] Y. Maday, A.T. Patera, and G. Turinici. A priori convergence theory for reduced-basis approximations of single-parameter elliptic partial differential equations. Journal of Scientific Computing, 17(1-4):437446, December 2002.

[14] C. G. Markidakis and I. Babuska. On the stability of the discontinuous galerkin method for the heat equation. SIAM Journal of Numerical Analysis, 34(1):389-401, 1997.

[15] A. K. Noor and J. M. Peters. Reduced basis technique for nonlinear analysis of structures. AIAA Journal, 18(4):455-462, April 1980.

[16] C. Prud'homme, D. Rovas, K. Veroy, Y. Maday, A.T. Patera, and G. Turinici. Reliable real-time solution of parametrized partial differential equations: Reduced-basis output bounds methods. Journal of Fluids Engineering, 124(1):70-80, March 2002.

[17] A. Quarteroni and A. Valli. Numerical Approximation of Partial Differential Equations. Springer, 2nd edition, 1997.

[18] W.C. Rheinboldt. On the theory and error estimation of the reduced basis method for multi-parameter problems. Nonlinear Analysis, Theory, Methods and Applications, 21(11):849-858, 1993.

[19] D.V. Rovas. Reduced-Basis Output Bound Methods for Partial Differential Equations. PhD thesis, Massachusetts Institute of Technology, 2002.

[20] Dominik Schötzau and Christoph Schwab. Time discretization of parabolic problems by the hp-version of the discontinuous galerkin finite element method. SIAM Journal of Numerical Analysis, 38(3):837-875, 2000 .

[21] Vidar Thomee. Galerkin Finite Element Methods for Parabolic Problems, chapter The Discontinuous Galerkin Time Stepping Method, pages 181-208. Springer Series in Computational Mathematics. Springer, June 1997.

[22] K. Veroy, D. Rovas, and A.T. Patera. A posteriori error estimation for reduced-basis approximation of parametrized elliptic coercive partial differential equations: "convex inverse" bound conditioners. Control, Optimisation and Calculus of Variations, 8:1007-1028, June 2002. Special Volume: A tribute to JL Lions. 